\title{
Congenital reflex myoclonus in two Merino-cross lambs in South Africa
}

D.J.C. Blignaut BVSc ${ }^{1 *}$, D.E. Holm BVSc, MSc(Vet) ${ }^{1}$, MRCVS, R. Leask BSc(Agric), BVSc, MMedVet(CaprOv) $^{1}$, N. Stander BVSc, MMedVet(DiagIm) $)^{4}$, J.C.A. Steyl BVSc ${ }^{3}$

${ }^{1}$ Department of Production Animal Studies, , ${ }^{3}$ Section of Pathology, ${ }^{4}$ Department of Companion Animal Clinical Studies, Faculty of Veterinary Science, University of Pretoria, Private Bag X04, Onderstepoort, 0110, South Africa

*Author for correspondence: dawie.blignaut@up.ac.za, +27125298154

Reflex myoclonic conditions have been reported in mice, Labrador Retriever dogs (March and others, 1993) Peruvian Paso horses (Gundlach and others, 1993) and Hereford calves (Harper and others 1986). The primary cause of myoclonus in these reports was consistent with a deficit in glycine receptors in the central nervous system. Similar conditions based on clinical findings and the lack of pathological lesions have not been reported in sheep.

Two, three day old, female Dohne Merino x Merino lambs from different ewes (assigned "Lamb 1" and "Lamb 2" for identification purposes) were presented with a complaint being unable to stand, having tremors and showing signs of stiffness. The flock is managed as a multisire breeding system. Annual vaccinations include: pulpy kidney, blue tongue, blue udder and Chlamydophila. Only these lambs were affected. Both lambs received sufficient colostrum from their respective dams shortly after they were born.

A complete clinical examination, neurological examination, bloodsmear for light microscopy evaluation, urinalysis and faecal analysis was performed. Both lambs appeared to be alert, could lift their heads and had no visual or auditory abnormalities. The lambs were able to suckle normally when assisted, but only when left in lateral recumbency. The lambs were recumbent and unable to rise or stand on their own and showed limited voluntary movement of front and hind limbs. Attempts to make the lambs stand only induced the typical whole body rigidity with the head and neck extended and characteristic hind leg adduction to crossing-over in a rigid extension manner (as seen by Blood and Gay 1971, Harper and others 1986, Windsor, personal communication, 2010). These myoclonic episodes were consistently characterised by rigidity of the body, extensor spasms, clearly palpable tachycardia and temporary apnoea. When returned to lateral recumbency both lambs resumed the initial posture, spasms ceased and normal respiration resumed after a short period of open mouth breathing. Sound stimuli in the form of sudden loud noises and sensory stimuli in the form of touch produced a milder response. Repetition of a sound stimulus (such as clapping the hands) lessened the intensity of the response until there was no response. Withdrawal reflexes in the front and hind legs were present, indicating intact spinal cord segments $\mathrm{C} 6$ through $\mathrm{T} 2$ in the front legs and the radial nerve and spinal cord segments L5 through S1 in the hind legs and sciatic nerve (as observed by Constable 
2004). Correction of the abnormal position during knuckling of the distal limbs seemed to be delayed, but it is believed that the whole body rigidity during manipulation obscured this procedure. Lamb 2 could stand for a short period with assistance after repeated attempts and was able to support her own weight while the whole body rigidity subsided momentarily, until she was stimulated again.

Whole blood in EDTA and serum were collected for haematology and serum concentrations of sodium, potassium, urea, creatinine, total calcium, magnesium, inorganic phosphorous, aspartate transferase, creatine kinase and bile acids. Abnormalities detected are summarised in table 1. All other clinical chemistry results for both lambs were within the normal reference ranges. The mildly elevated serum creatinine kinase, serum urea and blood platelet count were considered to be of less significance to the diagnosis of this particular case.

Cisternal cerebrospinal fluid samples were collected and analysed. The lambs were sedated with $0.5 \mathrm{mg}$ midazolam (Dormicum ${ }^{\circledR}$, Roche Products) and 20mg ketamine (Ketamine-Fresenius, Bodene). Gross appearance of both samples was clear and colourless. Analysis of the specific gravity, red cell count, white cell count and protein concentration were within normal reference ranges (Scott, 2010).

Extended ventrodorsal pelvic radiographs of each lamb were taken. Coxofemoral abnormalities were ruled out. The consistent hip joint lesions that were seen in polled Hereford calves with inherited congenital myoclonus (ICM) were not observed radiographically. It was suggested that the hip joint lesions seen in the calves with ICM were secondary as a result of severe myoclonic contractions of the pelvic adductor muscles seen by extensor crossing of the hind limbs (Harper and others 1986). It is possible that these sheep are traditionally selected for wool and not muscle mass, therefore the possibility of having a relative smaller adductor muscle mass might influence the frequency at which these distinctive hip joint lesions were seen.

Computed tomography (CT) images of the neurocranium of Lamb 1 were taken to investigate any gross structural abnormalities and cerebellar hypoplasia ante mortem. There were no visible gross structural abnormalities and the cerebellum was of normal size.

Lamb 1 was treated with $10.2 \mathrm{mg}$ vitamin E acetate and 0.4mg selenium (VitESe Injection, Kyron Laboratories). The neurological signs present and lack of improvement in clinical signs after treatment, excluded any deficiencies in these elements and was not investigated further.

After a period of two weeks the lambs were humanely euthanased with intravenous overdose of pentobarbitone and sent for post mortem examination. No significant pathological lesions could be demonstrated in any tissues on routine macroscopical or histological examination. There were no 
abnormalities in the hip joints of both lambs. Long term nursing of the recumbent lambs was complicated by dermal pressure injuries. The lack of pathological lesions at macroscopic and histological level ruled out a number of differential diagnoses affecting the neuromuscular and skeletal systems in lambs.

A specific aetiology for the condition could not be demonstrated with routine diagnostic procedures. A presumptive diagnosis of congenital reflex myoclonus was made. The diagnosis is based on clinical similarities between other animal models previously studied that showed reflex myoclonus upon stimulation. To investigate the possibility of a molecular neuromuscular cause, further specific genetic and immunohistochemistry analysis would be required to study the condition seen in these Merinocross lambs.

\section{Acknowledgements}

The authors acknowledge the Onderstepoort Veterinary Academic Hospital, the Department of Production Animal Studies and the Veterinary Genetics Laboratory, University of Pretoria, for financial support, as well as Miss Kate Moseley and other final year veterinary students who cared for the lambs during hospitalisation. Special thanks to Dr C.K. Harper (Veterinary Genetics Laboratory) for her efforts with the genetic analysis.

\section{References}

BLOOD, D.C. \& GAY, C.C. (1971) Hereditary neuraxial oedema of calves. Australian Veterinary Journal 47, 520

CONSTABLE, P.D. (2004) Clinical examination of the ruminant nervous system. Veterinary Clinics of North America - Food Animal Practice 20, 185-214.

GUNDLACH, A.L., KORTZ, G., BURAZIN, T.C.D., MADIGAN, J. \& HIGGINS, R.J. (1993) Deficit of inhibitory glycine receptors in spinal cord from Reruvian Pasos: evidence of an equine form of inherited myoclonus. Brain Research 628, 263-270

HARPER, P.A.W., HEALY, P.J. \& DENNIS, J.A. (1986) Inherited congenital myoclonus of polled Hereford calves (so-called neuraxial oedema): a clinical, pathological and biochemical study. Veterinary Record 119, 59-62

MARCH, P.A., KNOWLES, K., THALHAMMER, J.G. (1993) Reflex myoclonus in two Labrador Retriever littermates: a clinical, electrophysiological and pathological study. Progress in Veterinary Neurology 4, 19-24.

PIERCE, K.D., HANDFORD, C.A., MORRIS, R., VAFA, B., DENNIS, J., HEALY, P.J., SCHOFIELD, P.R. (2001) A Nonsense mutation in the $\alpha 1$ subunit of the inhibitory glycine receptor associated with bovine myoclonus. Molecular and Cellular Neuroscience 17, 354-363. 
SCOTT, P.R. (2010) Cerebrospinal fluid collection and analysis in suspected sheep neurological disease. Small Ruminant Research doi: 10.1016/j.smallrumres.2010.04.009

Table 1: Comparative abnormalities observed between Lamb 1 and Lamb 2.

\begin{tabular}{llll} 
& Lamb 1 & Lamb 2 & Reference values \\
\hline Identification number & $05 / 218$ & PU03204 & \\
Severity of myoclonic spasms & More severe & Less severe & \\
Computed Tomography (CT) findings & Normal & Not done & \\
Response to treatment with VitE/Se & No response & Not treated & \\
Serum Creatinine Kinase & $110 U / 1$ & $61 \mathrm{U} / 1$ & $12-51 \mathrm{U} / 1$ \\
Serum Urea & $7.50 \mathrm{mmol} / 1$ & $6.20 \mathrm{~mol} / 1$ & $2.65-2.64 \mathrm{mmol} / 1$ \\
Serum Creatinine & $73 \mu \mathrm{mol} / 1$ & $40 \mu \mathrm{mol} / 1$ & $44-150 \mu \mathrm{mol} / 1$ \\
Blood neutrophil count & $4.35 \times 10^{9} / 1$ & $5.71 \times 10^{9} / 1$ & $0.40-5.00 \times 10^{9} / 1$ \\
Blood platelet count & $1505 \times 10^{9} / 1$ & $2317 \times 10^{9} / 1$ & $250-750 \times 10^{9} / 1$ \\
\hline
\end{tabular}

Figure 1: Lamb showing myoclonic whole body rigidity with cross-over extension of hind limbs.

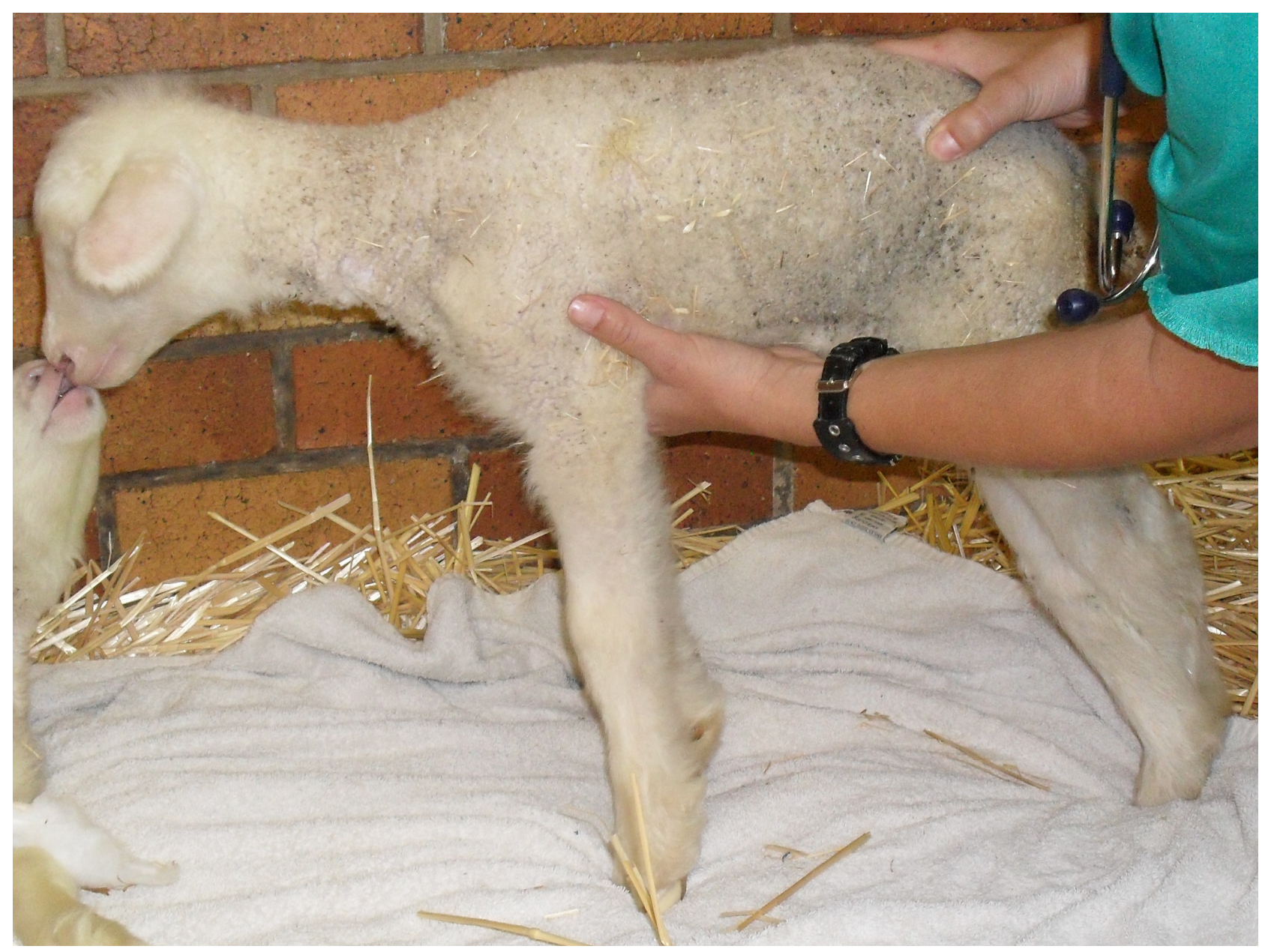

